\title{
Die Anästhesie beim Hund mit Medetomidin, Ketamin und Diazepam
}

\author{
Nuh KILIÇ', Ali ERKUT ${ }^{2}$ \\ 'Chirurgische Abtcilung der Veterinärmedizinische Fakultät der Universität Adnan Menderes, Aydun: 2Aus dem . Amt für \\ Veterinärwesen der Stadtverwaltung Mamak, Ankara
}

\begin{abstract}
Zusammenfassung: In der vorliegender Arbeit wurde die Kombination von Medetomidin (Domitor(B)). Ketamin (Ketalar(B)-5()), und Diazepam (Diazcpam-ratiopharm(B) zur Anästhesie beim Hund hinsichtlich ihrer Wirkungen und Vebenwirkungen untersucht. Für das Versuchsvorhaben wurden insgesamt 10) weibliche Hunde der Rasse Deutsche Schäterhund cingesetzt. Vor der Anasshesic, alle 15 Minuten während der Anästhesic sowie 24 Stunden nach der Anästhesie wurde bei allen Tieren Blut entnommen. um lithordiagnostische (die Elektrolyte und das Blutbild) und klinisch-chemische (dic Leberwerte. Nicrenwerte) Untersuchungsparameter auszuwerten. Die Atemfrequenz. die Herzfregen\% und dic Körpertempelaltur während der Operation zeigt cin signifikanter Abfall ( $p<0) .(15)$ zum Ausgangswert. Auch die AST und Glukose erhöht sich auf signifiktnter Weise während der Anästhesic $(p<0.05)$ und 24 Stunden später nach der Anästhesie $(p<0.05)$. Die sedative Konpenenle der Medelomidin-Ketamin-Diazepam Kombination ist schr alusgeprägt. Alle Tiere sind ausgezeichnet relaxien und acigten keine Reatktionen atuf das schmerzhafte Manipulationen. Dic klinische und laborchemische Untersuchungen zeiglen. dalls dieser Art der Anasthesie beim Hund ohne Komplikationen durchzuführen ist.
\end{abstract}

Schlisselwörter: Allgemeinanisthesie, diazepam. hund. ketamin, medetomidin

\section{Köpeklerde medetomidin-ketamin-diazepam anestezisi}

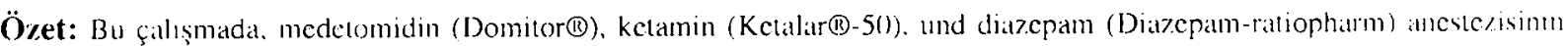

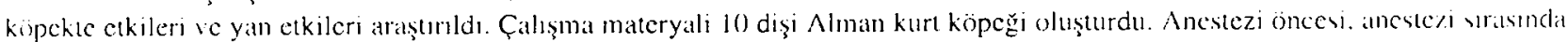
(her 15 dakikadat bir) ve ancstezi sonrasinda hayvanlardan kan örnekleri almarak klinik parametreler. hemogram ve bitzl kall serum değgerleri incelendi. Anestezi sirasinda solunum frekansi, kalp frekansi ve viecut isisinda anestezi öncesine nazaran al/alma ( $p<1)(1) 5)$ istatistiki aç)dan önemli bulunmuştur. Yine anestezi sırasinda ve anesteziden 24 saat sonra AST ve glukoz değerleri ancsteri önccsine naZzaran istatistiki bir artı̣ ( $p<0 .(05)$ göstermiștir. Medetomidin-kctamin-diazepam kombinasyonunun sedatil etkisi oldukçal geiç̨ii hulunmuştur. Tüim hayvanlarda mükenmel bir kas gevşemesi ve ağrıl maniplasyonlara karşı hayvaunlarda reaksiyon gösterınediği tespit edilmiştir. Sonuç olarak, bu kombinasyonun köpeklerde kullanılmasıyla komplikasyondan uzak hir anestezi

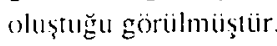

Anahtar kelimeler: Diazepam, gencl ancstezi, ketamin. köpek, medetomidin

\section{Einleitung}

Mcdetomidin gilt pharmokologisch als potenter, selekliver und spezifischer $\alpha_{2}$-Adrenorezeptor-Agonist sowohl des zentralen als auch des peripheren Nervensystems (19,28). Die Wirkung von Medetomidin tritt nach intravenöser. intramuskulärer und subkutaner Applikation ein, während cine orale Verabreichung wegen les starken Erste-Phase-Mctabolismus in der Leber nicht zum gewünschten Frfolg führt $(23,25,27)$. Durch Stimulation prä- und postsynaptischer $\alpha_{2}$ - $\Lambda$ drenorezeptoren im Zentralnervensystem soll die Substanz eine Sedation, Muskelrelaxation und Analgesic bewirken (26-28). Die durch Medetomidin bewirkte Anxiolyse, Sedation und Analgesie ist dosisabhängig und hält je nach Tierart zwischen einer und cincinhalb Stunden an. Bei höheren Dosierungen verlängert sich jedoch nur die Wirkdauer, aber nicht die Sedationstiefe $(15,19,21,25)$. Als optimale Dosierung für ausreichende Sedicrung, Analgesie und $\mathrm{Mu-}$ skelrelaxation beim Hund haben sich $40 \mu \mathrm{g} / \mathrm{kg}$ intramusküler oder intravenös erwiesen $(7,9,26,27)$. $\alpha_{2}$ - $\Lambda$ drenorezeptor-Agonisten wic Medetomidin hewirken durch dic Besctzung der Rezcptoren zentral ein Abnchmen des Sympathikotonus und eine Abnahme der Noradrenalina usschüttung aus postganglionären symphalischen Nervenendigungen sowie einen reflexartigen $\Lambda$ nstieg des $V$ algotonus. Dieses Zusammenspicl hewirkl cine Bradykardic bei anfänglicher Hypertension und peripherer Vasokonstriktion $(17,24)$. Neben der Bradykardie können nach Medetomidingabe unabhängig von der verabreichten Dosis in der Einleitungsphase der Narkose Herzrythmusstiorungen auftreten $(6,8,12)$.

Dosisabhängig bewirkt Medetomidin sowohl nach intramuskülärer als auch nach intravenöser Applikation 
eine Atemdepression (4,5). Durch Becinflussung 7entraler noradrenerger Mechanismen in Hypothalamus bewirken $\alpha_{z}$-Adrenorezeptor-Agonisten wic Medetomidin cine Hypothernie (28).

Ketamin führt zum Zustand der dissoziativen Anasthesic, die durch Analgesie, oberfläclichen Schlaf und Kalalepsie gekennzeichnet ist. Das ZNS wird funktionell gespalten. Wahrscheinlich dämpft es das thalamokorlikale Zentrum, während das retikulumaktivierende und das lymbische System stimuliert werden. Diese Stimulalion kann zu Anfällen führen $(5,18,22)$. Das Ketamin wird der zentrale Sympathikotonus gesteigert. Dies führt 7u cinem Anstieg der Herzfrequenz, des Herzminutenvolumen, des mittleren arteriellen Druckes $(10,16,20,22)$. Nach intravenöser Gabe von $10 \mathrm{mg} / \mathrm{kg} \mathrm{Kc}$ talmin heim Hund werden Atemfrequenz und Atemminutenvolumen gesenk1, während das Atemzugvolümen unverändert bleibt (10.20). Ketamin führt bei ausreichend hoher Dosicrung 7.u gesteigertem Muskeltonus, Husten-, Schluck- und Lidreflex bleiben erhalten. Es wird sowohl Hypothermie durch Dämpfung des Thermoregulationszentrums, als auch Hyperthermic als Folge der gesteigerten Muskelaktivität festgestellt. Für Ketamm steht derzeil kein zuverlässiger Antagonist zur Verfügung (2. 10,14,18,22).

Diazepam gehört zur Gruppe der Benzodiazepine und wirken nach Löscher (18) dosisabhängig anxiolytisch, antikonvulsiv, antiaggressiv, sedicrend, hypnotisch und zentral muskelrelaxierend. Diazepam hemm im Rückenmark polysynaptische Reflexe. Dies löst cine zentrale Muskelrelaxation aus. In Kombination mil anderen, zentral dämpfenden Pharmaka kann jedoch cine chirurgische Toleranz crzielt werden. Die Wirkung von Diazcpam auf Herz und Kreislauf ist minimal. Dic Kontraktilital des Myokards wird leicht verringert, der Blutdruck wird unbedeutend gesenkt. Dic Herzfrequenz. bleibt unverändert oder steigt leicht an $(3,16)$ Diazepan übt nur geringen Einlluss auf die Atmung aus. Atemfrequenz, Atemzugvolumen und Atemminutenvolumen ändern sich nur geringfügig $(1,11,13)$. Wird ein Überhang der Benzodiazepinwirkung beobachtet, kann dieser und mit der Gabe des spezifischen Benzodiazepinantagonisten Flumezanil und Sarmazenil aufgehoben werden $(2,13)$.

7icl der vorliegenden Arbeit soll es sein, dic Auswirkungen von der Kombinationsanästhesie bein Hund mit Medetomidin, Ketamin und Diarepam auf einigen physiologischen Funktionen und auf den Blutparameter zu crmitteln.

\section{Material und Methoden}

Für das Versuchsvorhaben wurden insgesam 10) Hunde der Rasse Deutsche Schäferhund, die in dic Klinik in der Ausbildungsschule für dic Militärische Tierärtze in Gemlik-Türkei für die Ovariohysterektomic cinzuliefern waren, cingesetzt

12 Stunden vor der Operation wurden die Tiere in Einzelboxen aufgestallt und nüchtem gesctzt. Am Tag der Operation wurden die Tiere mil Medelomiclin (Domi tor $\left.{ }^{\circledR}\right)$ in der Dosicrung $40 \mu \mathrm{g} / \mathrm{kg}$ intramuskülär prä. medizicrt. In ausreichender Sedation fixicrt man eine Venenverweilkatheter in der $\mathrm{V}$. cephalica antebrachium und injizierte Ketamin hidroklorür (Ketalar@-50) $10 \mathrm{mg} / \mathrm{kg}$ und Diazcpam (Diazepam-ratiopharm®) $0,5 \mathrm{mlg} / \mathrm{kg}$ intravenös zur Fortführung der Anästhesic. Vor der Anästhesie, alle 15 Minuten während der Anästhesic sowie 24 Stunden nach der Anästhesic wurde bei allen Tieren Blut entnommen, um labordiagnostische (dic Flektrolyte und das Blutbild) und klinisch-chemische (die Leberwerte, Nicrenwerte) Untersuchungsparameter auszuwerten. Das Blutbild wird von einem Analysengerät (Coulter STKS9) ermittelt. Für die serologische Untersuchungen wurde ein anderes Meßsgerial(Technicon RA $1000)$ benutzt.

Die rechnerische Aufbereitung des Datenmaterials erfolgt mil dem Statistikprogrammpaket SPSSC (Statistical Package for the Social Science, Version 10.0). I ür alle untersuchten Parameter werden der arithmetische Mittelwert, der Medianwert und die Standardfehler berechnet. Unterschiede an den cinzelnen Mckparameter werden mit Hilfe von t-Test für abhängjge Stichproben errechnet.

\section{Ergebnisse}

Nach der Verabreichung von Medelomidin wurde bei allen Tieren eine mittel- bis hoch gradige Sedation beobachtet. Die Ketamin-Diazepam Kombination bewirkte bei allen Tieren die Ausbildung einer Anästhesic. dic ungefähr eine Stunde dauerte. Während der Operation be obachtete man eine gute Analgesie und Muskelrelaxation. In der Aufwachphase und nach der Anäshesie erholen sich alle Tieren ohne Komplikationen. Die Alemfrequenz, die Herzfreqenz und die Körpertemperalur wïhrend der Operation zeigl ein signifikanter Abfall $(p<0.05)$ zum Ausgangswert. Dic Leukozyten steigt am Ende der Operation und 24 Stunden nach der Operation hochsignifikant $(\mathrm{p}<0.01)$ an. Im Vergleich zum Vorwert zejgten dic Werte von Thrombozylen und Hämalokrit. Natrium und Kalium während der Anästhesic ein signifikanter Ansticg $(\mathrm{p}<0.05)$ in Referenzbereich. Gleichfalls ist bei der Kre- 
atininwertc während der Anästhesie cin signifikanter Anstieg $(p<0.05)$ zu beobachten. dic aber im physiologischen Referenzbereich liegen. Auch dic AST und Glukose crhöht sich auf signifikanter Weise während der Anästhesie $(\mathrm{p}<0.05)$ und 24 Stunden später nach der Anästhesie $(p<0.05)($ Tabelle 1$)$.

\section{Diskussion}

Der Einfluss der Medetomidin/Ketamin-Kombination aul die Herzfrequenz ist günstig zu beurteilen. Nach der Applikation von Medetomidin/Ketamin tritt der Abfall der initial erhöhten Herzfrequenz in phyisiologische Bereiche auf. Der signifikante Pulsfrequenzabfall während der Narkose ist zu crwarten, nach dem $\alpha_{2}$ Adrenorezeptoragonisten eine bradykardie Wirkung haben $(4,6,8,12,24,27)$. Das signifikante Absinken entspricht den Iirfahrungen vieler Autoren. Day und Muir (8), Ruffalo (24) und Vainio und Palmu (27) erklären dies u.a. mit ciner zentral gesteuerten Abnahme des Sympathikotonus und einem reflexartigen Anstieg des Vago. tonus. Moens und Forgetton (19) sehen die durch Medetomidin induzierte Bradykardie als Folge der starken Vasokonstriktion, dic über die starke Reizung des $\mathrm{Ba}$ rorezepterrellexes ausgelöst wird. Trotz dieser starken Becinflussung des Kreislaufsystems durch Medetonidin ercigneten sich in der vorliegender Studie keinerlei klinisch relevanten $Z$ wischenfälle.

Der signifikante Abfall der Körpertemperatur während der Anästhesic ist durch Erregung zentraler $\alpha_{-}-$ Adrenorezeptoren in Hypothalamus bedingt (5) und erstreckt sich ïber längeren 7citraum. Dic Körpertemperatur während der Anästhesie und auch währond der Aufwachphase sollte in regelmässigen Ahständen uberprüft werden, um gegebenfalls cinem $z$ slarken $A$ biall entgegenwirken zu können $(25,26)$.

Die Nebenwirkungen der Medetonidin/KetaminKombination auf das respiratorische Systen sind ausgeprägt $(6,7,15,24)$. Dies kann jedoch nicht aus dem Abfall der Atemfrequenz geschlossen werden. Dieser resultient aus ciner präanästhetisch erhöhten Alemfrequenz aufgrund der Aufregung der Tiere. Sowohl der Ablall der Atemifrequen $z$ als auch der $\Lambda$ bfall der Sauerstof fättigung während der Beobachtungszeitraumes lassen auf die Ausbildung einer milderen Atemdepression schlicssen.

Leukozytenwerte bleiben am Ende der Anästhesic und in den früpostoperativen Phasen hochsignifikant über den Vorwert. Für diesen Ansticg kann die ent 7ündliche Reaktion an der Operationswunde sein.

Für den signifikanten Anstieg der Thrombozylenzahl während der Operation könnte die akute Blutungen der Grund sein

Tabelle 1. Tabellarische Darstellung der klinischen Parameter, des Blutbildes sowic der klinisch-chemischen Laburparimeler im Blutserum vor der Anästhesic und während der Anästhesic sowic nach der Anästhesic (x=Mittclwert. Sx=Standardichter)

\begin{tabular}{|c|c|c|c|c|c|}
\hline Phatse & $\begin{array}{l}\text { () (Vorwert) } \\
x \pm S x\end{array}$ & $\begin{array}{l}\text { 15.Minute } \\
x \pm S x\end{array}$ & $\begin{array}{l}\text { 30. Minute } \\
\mathrm{x} \pm \mathrm{Sx}\end{array}$ & $\begin{array}{l}\text { 45.Minule } \\
x \pm S x\end{array}$ & $\begin{array}{l}2+\text { Sullude } \\
x \pm S x\end{array}$ \\
\hline Herzfrequenz(min'l) & $94,2+4,23$ & $76.6+3.20 *$ & $70,7+4,57 *$ & $74,8+5.47 *$ & $101.6+3.38$ \\
\hline Atcmfrequenz $\left(\mathrm{min}^{-1}\right)$ & $32,6+2,58$ & $24.6+3.58 *$ & $25.2+1.95^{*}$ & $26.8+1.59: 5$ & $33.1+1.79$ \\
\hline Körpertemperatur $\left({ }^{\circ} \mathrm{C}\right)$ & $38.65+0,05$ & $38.15+0.08^{*}$ & $38,20+0,07 *$ & $38,30+0.06 \%$ & $38.89+1) .03$ \\
\hline L.cukozytenzahl $\left(106 / \mathrm{mm}^{3}\right)$ & $12,78+10,78$ & $13,39+2.73$ & $12.8+2.33$ & $16.9+3.2()^{* *}$ & $26.4+2.5 .3 *$ \\
\hline Frythrozytenzathl $\left.(10)^{3} / \mathrm{mm}^{3}\right)$ & $7.65+1.96$ & $8,03+2,46$ & $7.43+1.83$ & $6.89+1.43^{*}$ & $7.77+1.6 .3$ \\
\hline Trombozycenzahl $\left(10^{3} / \mathrm{mm}^{3}\right)$ & $497,5+63,2$ & $659,9+49.8^{*}$ & $646.2+19.2^{*}$ & $617.7+64.5$ & $0.37 .8+51.4$ \\
\hline Hämoglobin(g/dl) & $20.67+0.56$ & $21,6+0.48$ & $19.1+0.32$ & $17.5+0.46$ & $18.8+4.311$ \\
\hline Hämatlokrit(\%) & $45,8+3,89$ & $54.6+1.31)^{*}$ & $49.0+1.27$ & $43.6+1.98$ & $49.11+12.5$ \\
\hline Natritum (mmol/l) & $131.3+2.37$ & $129+4.56$ & $139,()+3.2 *$ & $136.5+4.033^{*}$ & $136.2+3.20$ \\
\hline Kalium (Inmol/l) & $4,34+0,17$ & $4,16+1), 13$ & $4.59+1.24$ & $4.89+1) .16^{\circ}$ & $4.49+0.21$ \\
\hline Harnstolf $(\mathrm{mg} / \mathrm{dl})$ & $24.5+2.53$ & $26.3+2.25$ & $28,2+2.54$ & $26.2+2.82$ & $26.2+1.16$ \\
\hline Kreatimin (mg/dl) & $1,03+0,05$ & $1.20+0.05 \%$ & $1.18+0,046$ & $1.21+11.08 \%$ & $1.06+0.015$ \\
\hline Albumin(g/dl) & $3,29+0.01$ & $3.32+0.01$ & $3.15+0.06$ & $3.39+0.01$ & $3.33+0.1) 1$ \\
\hline Gesilmtprotein(g/dI) & $5.73+0.22$ & $5.78+(0.02$ & $5.68+2.19$ & $5.63+10.02$ & $5.69+0.012$ \\
\hline Glucose $(\mathrm{mg} / \mathrm{dl})$ & $87,2+4,98$ & $82,1+2,78$ & $97,7+3,43 *$ & $92.5+3.04 \%$ & $101.7+2.87:$ \\
\hline $\mathrm{AST}(\mathrm{L} / \mathrm{L})$ & $45,4+6,37$ & $39.1+4,94$ & $62,5+4,53 *$ & $58.1+6.22$ & $66.7+3.88 \%$ \\
\hline$\wedge L T(L / L)$ & $29,8+3.15$ & $29,9+4,71$ & $35,5+3,13$ & $32,3+2,35$ & $4(1.2+5.012 *$ \\
\hline
\end{tabular}

“ $p<0.05$ (Irrtumwahrscheinlichkcit $<5 \%$, signifikant) signifikanter Unterschied zum Vorwert

:* $p<(),()]$ (Irrtumw'ahrscheinlichkcit $<1 \%$,hochsignifikant) signifikanter Lnterschied zum Vorwert 
Die während der Operation verabreichten hypertonen Kochsalzlösungen dürfte für den signifikanten $\mathrm{An}$ sticg der Natriumwerte (Hypernatriänie) verantwollich sein.

Harnstoff wird im Harnstoffzyklus in der Leber aus Ammoniak synthetisiert. Er kann als das entgiftete Stoffwechselendprodukt der Proteinverdauung betrachtet werden, das normalerweise über die Niere ausgeschieden wird. Der Im Blut hestimmle Wert ist daher nahrunsabhaingig. Kreatinin ist ein Produkt des endogenen Muskelstoffwechsels. Es wird gebildet aus Kreatin und Phosphokreatin. Dic Serumkonzentration steht in gewissen Bezichung zur Muskelmasse des Individiums. Kreatinin hat gegeniber dem Harnstoff den Vorteil, daß es nicht nahrungsabhängig isl und auch vom endogenen Protcinmelabolismus nicht becinflußt wird (14). Auch dic Kreatininwerte steigen in unserer Arbeit signifikant. Aber die Werte liegen im oberen Referenzbereich.

Die Aspartat-Amino-Transferase (AST) kann in unkerschiedlicher Aktivität in zahlreicher Gewebe und Organen angetroffen werden. Sie ist also keineswegs organspezifisch. Aktivitätsstörungen his zum Dreifachen kïnnen als leichte Erhöhungen, über das Dreifache als schwere Aktivitätssteigerungen bezeichnel werden. Ausser hei Leberkrankheiten sind AST-Aktivitäten bcsonders auch bei Muskelkrankheiten erhöht. Man wird daher nie die AST als cinziges Enzym bestimmen (14). Einc leichle Erhöhung der AST in der vorlicgender Arbeit kounnte an der Muskel\%uckungen während der Operation licgen.

Dic sedative Kompenente der Medetomidin/ Kctamin-Kombination ist sehr ausgeprägt. Alle Tiere sind ausgezeichnet relaxiert und zeigten keine Reaktionen auf das schmerzhafte Manipulationen. Dic Kombination Medetomidin-Diazepan zeigte sich als eine sehr angenehm zu handhabende Narkose für längere, schmerzhafte Eingriffe. Wic Alef und Schmidt-Oechterning (2) beschrieben, konnte auch hier beobachtet werden, daB Diarepan die analgetische Kompenente des Medetomidin zu vermag

Trotz der nicht unerheblichen Belastungen des Atem- und Kreislaufsystems der verwendeten Medetomidinkombination trat in der vorliegender Studic keine klinisch relevanten Zwischenfälle bei der Narkose auf. Die Nebenwirkungen auf das Krcislaufsysten lassen sich durch eine anticholinerge Prämedikation abschwiachen. Atropin wirkt der Bradykardie und der Ausbildung von Sinusarrythmien und $A V$-Blöcken unter $M c$ detomidineinfluss entgegen (4,23). Aufgrund dieser
Kreislaufdepression solle Medetonidin beim her\% kranken Hund nicht eingesetzt werden (15.17).

\section{I iteratur}

1. Alef M, Schmidt-Oechterning GL (1993): Injektionsunaesthesie für kurze Eingriffe. 34-45. In: GU SchmidtOcchterning, $M$ Alef (Hrsg). Neue Aspektc der Vetcrinacranacstesic und Intensivtherapic. Patul Parcy Verlag. Berlin.

2. Alef M, Schmidt-Oechterning GL (1993): Antersmisiertbute Anaesthesie. 75-82. In: GLi Schmidt Oechterning. M Ald (Hrsg), Neuc Aspekte der Vecrinaerinalestesic und Intensivtherapic. Paul Parey Verlag. Berlin.

3. Balderssani RJ (1985): Benzodicisepines. 433-437. In: GA Goodman, GS Goodman (Eds). The Pharmacological Basis of Therapeutics. Macmillan Publishing Company. New York.

4. Bergström K (1988): Cardiovascular and pulmomare of fects of a new sedativanalgesic (medetrmidin) as a pre ancaesthetic drug in the dog. Acta Vet Scind, 29. $109-116$

5. Büch HP, Büch U (1992): Narkotika. 232-253. In: W Forth. D Henschler. W Rummel (Hrsig). Allgemeine und Spericlle Pharmakologic und Toxikologic. B.I. Wissenschalsverlag, Mannheim.

6. Clark KW, England GCW (1989): Medetomidin, a new sedative-analgesic for use in the dong and its reversal wath atipemazol. J Sinall Anim Pract, 30. 343-348.

7. Coper H, Helmchen H (1992): Psychopharmaka. 281 . 303. In: W Forth. D Henschler. W Rummel (Hrsig). All gemeine und Spezielle Pharmakologic und Toxikologic B.I. Wissenschafsverlag, Mannheim.

8. Day TH, Muir WW (1993): Alpha 2-adrentergic receptot agonist effects on supraventricular and rentricular at tomaticity in dogs with complete atrinentricular black Am J Vet Res. 54. 136-141.

9. Hamlin RL, Bednarski IS (1989): Studies to determme the optimal dose of Medetomidine for the dos. Acta $\mathrm{Val}$ Scan. 85, 89-95.

10. Haskins SC, Farver TB, Pat\% J1) (1985): Ketcumin in dogs. Am J Vet Res. 46. 1855-1800).

11. Haskins SC, Farver TR, Patz JD (1986): Cordion'cascular changes in dogs given diazepam and diazepan-ketamine. Am J Vet Res, 47, 795-798.

12. Hayashi $K$, Nishimura $R$, Yamaki A, Matsugana $S$ (1995): Cardiopulmonan effects of medetomadine. mede'to. midine-midazolam and medetomidine-midaroskan-altpame-ste in dog.s. J Vel Med Sci, 57. 99-1(14.

13. Hunkeler W, Möhler H, Piere L, Polc P, Bonett EP, Cumin R (1981): Selective antagonist of henzodiazepines Nature. 290. 514-516.

14. Kraft W, Dürr UM (1995): Klinische Lshordiagnosilk fur die Tiermedizin. Schattatuer. Sultgart

15. Kramer S, Nolte I, Pöttman B, Jöchle W (199?) Z Zu analgetischen Wirkung des alpha 2-Simpathomimesikum. Medetomidin heim Hund. Kleinlicrpraxis. 38. 5- 12

16. Larsen R (1990): Anciesthesie. Urban und Schwarzenberg. München. 
17. Lombard CW, Kwart C, Sacteri (i, Holm G (1989): liffects of medetomidine in degs with mitral regurgitation. Acla $V_{c 1}$ Scan, 85. 167-174

18. Lïscher W, Ungemach FR, Kroker R (1994): Cirundlagen der Pharmakosherapie bei Haus- und Nutzlieren. Paul Parcy. Muinchen.

19. Voens T, Forgetton X (1990): A comparative study of mede'omidine/ketamin and rylasinketamin anaesthesie in dog.s Vet Rec. 127, 567-571.

20). Muir WW, Hubbel JAE (1988): Cardinpulmonary and anesthetic effects of ketamine and its enantiomers in dogs. Am J Vet Res, 49. 530-534.

21. Vilsfors L, Garner L, Adofsson A (1989): Sedative and cinalgesic effects of medetomidine in dogs-an open clinical sluds: Acla Vel Scand, 85, 155-159.

22. Paddleford RR, Echardt W (1992): Anciesthesie bei Kleintieren. Schatcuer, Stutgart.

23. Racihae JE, Racihac MP, Short CE (1989): Me detomidine as a preanesthetic: prior to ketanin-HCL and halothan anesthesia in laboratory beagles. Acta Vet Scan. 85. $111-116$.
24. Ruffalo RR (1985): Distribution and function of peripheral alpha-adrenorecepors in the cardiovacular system. Pharm Biochem Behav, 22. 827-833.

25. Salonen JS (1989): Pharmakokinetics of medetomidine ^cta Vel Scan. 85. 49-54.

26. Vache-Vache $\mathbf{T}$ (1989): Clinical erellection of the detomidine a novel sedative and andisesic drus for dess: and cats. Actat Vet Scan, 85. 151.153.

27. Vainio O, Palmu L (1989): Cardiorascular and respiraton effects of medetomidine in dogs and influence of anticholinergics. Acta Vet Scan. 30. 401-4018.

28. Virtanen R (1989): Pharmalogical profiles of medetomidine and its antagonist, atipemazole. Acta Vet Scan. 85. 29-37.

Eingegangen am 15 Oktober $2001 /$

Akzeptiert am 28 Dezember 2001

\section{Korrespondenzadresse:}

Dr. vet.med. Nuh Kılı̧

Adnan Menderes Universitesi

Veteriner Fakultesi

Cerrahi Anabilim Dalt

Işkll Kïyü Bitisiği

Ban Kampuisui- Aldun 